Research

Open Access

\title{
Decrease of CD4-lymphocytes and apoptosis of CD14-monocytes are characteristic alterations in sepsis caused by ventilator-associated pneumonia: results from an observational study
}

Aimilia Pelekanou${ }^{1}$, Iraklis Tsangaris ${ }^{2}$, Antigoni Kotsaki ${ }^{1}$, Vassiliki Karagianni ${ }^{1}$, Helen Giamarellou ${ }^{1}$, Apostolos Armaganidis ${ }^{2}$ and Evangelos J Giamarellos-Bourboulis ${ }^{1}$

\author{
14th Department of Internal Medicine, ATTIKON University Hospital, 1 Rimini Str., Athens 124 62, Greece \\ ${ }^{2} 2$ ndDepartment of Critical Care, ATTIKON University Hospital, 1 Rimini Str., Athens 124 62, Greece
}

Corresponding author: Aimilia Pelekanou, aimpelekanou@yahoo.gr

Received: 19 Aug 2009 Revisions requested: 12 Oct 2009 Revisions received: 22 Oct 2009 Accepted: 2 Nov 2009 Published: 2 Nov 2009

Critical Care 2009, 13:R172 (doi:10.1186/cc8148)

This article is online at: http://ccforum.com/content/13/6/R172

(c) 2009 Pelekanou et al.; licensee BioMed Central Ltd.

This is an open access article distributed under the terms of the Creative Commons Attribution License (http://creativecommons.org/licenses/by/2.0), which permits unrestricted use, distribution, and reproduction in any medium, provided the original work is properly cited.

\begin{abstract}
Introduction The present study aimed to investigate changes of the immune response between sepsis due to ventilatorassociated pneumonia (VAP) and sepsis due to other types of infections.

Methods Peripheral venous blood was sampled from 68 patients with sepsis within 24 hours of diagnosis; 36 suffered from VAP; 32 from other nosocomial infections, all well-matched for severity, age and sex. Blood monocytes were isolated and cultured with/without purified endotoxin (lipopolysaccharide (LPS)). Estimation of tumour necrosis factor alpha (TNF $\alpha)$ and interleukin-6 (IL-6) in cultures' supernatants was done by an enzyme immunoassay. Flow cytometry was used to determine subpopulations of mononuclear cells and apoptosis. To mimic pathogenesis of VAP, mononuclear cells of healthy volunteers
\end{abstract}

were progressively stimulated with increased inocula of pathogens; apoptosis was determined.

Results In patients with VAP, the absolute number of CD3(+)/ CD4(+) lymphocytes was significantly lower $(P=0.034)$ and apoptosis of isolated monocytes was increased $(P=0.007)$ compared to other infections. TNF $\alpha$ and IL- 6 production from LPS-stimulated monocytes was lower in patients with VAPrelated sepsis than with sepsis due to other infections. Apoptosis of monocytes was induced after in vitro stimulation of mononuclear cells by a mechanism mimicking VAP.

Conclusions Decrease of CD4-lymphocytes and immunoparalysis of monocytes are characteristic alterations of sepsis arising in the field of VAP.

\section{Introduction}

Sepsis is an important cause of admission and mortality in intensive care units (ICU). In Europe, the Sepsis Occurrence in Acutely III Patients study disclosed an ICU mortality rate from sepsis ranging between $27 \%$ and $54 \%$ depending on the severity [1]. In the USA, 215,000 deaths are reported annually due to sepsis [2].
Ventilator associated pneumonia (VAP) is the most common nosocomial infection and the leading cause of sepsis in the ICU. Up to $28 \%$ of patients receiving mechanical ventilation will eventually develop VAP, with a mortality rate of up to $70 \%$ [3-7].

Various explanations have been proposed for the increased mortality of patients with VAP. One previous study from our group in a cohort of 90 patients with sepsis and VAP mainly 
caused by Gram-negative bacteria disclosed an association between derangements of the innate immune system and mortality. More precisely, patients with early monocyte apoptosis greater than $50 \%$ were less likely to die compared with those exhibiting monocyte apoptosis lower than 50\% [8]. However, it was not studied whether apoptosis of monocytes is the only detrimental alteration of the immune response linked to final outcome or if other changes of the adaptive immune system may have an effect as well. It should also be noted that this latter study was focused on patients with sepsis due to VAP, whereas sepsis of other infectious etiologies may differ in terms of its immune responses.

The present study was designed to unravel the unique features of the innate and adaptive immune responses of patients with sepsis due to VAP compared with patients with sepsis due to other infectious diseases and to propose a mechanism mediating these differences.

\section{Materials and methods Study population}

A total of 68 patients were enrolled in the study. Patients were hospitalized in the second Department of Critical Care Medicine and in the fourth Department of Internal Medicine of ATTIKON University Hospital in Athens. The study was approved by the Ethics Committee of the hospital. Written informed consent was provided by patients or their relatives. All patients were older than 18 years. Exclusion criteria included neutropenia ( $\leq 500$ neutrophils/ $\mu$ ), HIV infection or oral intake of corticosteroids at a dose equal to or higher than $1 \mathrm{mg} / \mathrm{kg}$ equivalent prednisone for at least one month.

All sequential admissions with sepsis, severe sepsis or septic shock were screened for enrolment during the period January 2006 to June 2007. Patients finally enrolled were those with septic syndrome due to VAP and those with septic syndrome caused by other types of infection, namely acute pyelonephritis, primary bacteremia, intraabdominal infection, communityacquired pneumonia (CAP) and hospital-acquired pneumonia (HAP), provided that they were well-matched to patients with VAP by age, sex, underlying conditions and disease severity.

Sepsis was defined as any microbiologically documented or clinically diagnosed infection accompanied by at least two of the following: core temperature above $38^{\circ} \mathrm{C}$ or below $36^{\circ} \mathrm{C}$; pulse rate above 90 beats/minute; respiratory rate above 20 breaths/minute or partial pressure of carbon dioxide (pCO2) below $32 \mathrm{mmHg}$; and leukocytosis (white blood cells (WBC) $>12,000 \mathrm{cells} / \mu \mathrm{l})$ or leukopenia $(\mathrm{WBC}<4000 \mathrm{cels} / \mu \mathrm{l})$ or presence of immature forms above $10 \%$ of total WBC count $[9,10]$.

Severe sepsis was defined as sepsis aggravated by the acute dysfunction of at least one organ. Acute organ dysfunction was defined as follows: acute respiratory distress syndrome, as any value of partial oxygen pressure/fraction of inspired oxygen (pO2/FiO2) less than 200 and diffuse bilateral infiltrations in chest X-ray; acute renal failure, as the production of less than $0.5 \mathrm{ml}$ urine/ $\mathrm{kg} /$ hour for at least two hours, provided that the negative fluid balance of the patient was corrected; metabolic acidosis, as any $\mathrm{pH}$ below 7.30 or any base deficit above $5 \mathrm{mEq} / \mathrm{l}$ and serum lactate at least more than twice the upper normal value; and acute coagulopathy, as any platelet count below 100,000 cells/ $\mu$ l or International Normalized Ratio above $1.5[9,10]$.

Septic shock was defined as sepsis accompanied by systolic arterial pressure lower than $90 \mathrm{mmHg}$ necessitating the administration of inotropic agents $[9,10]$.

Diagnosis of VAP was established if all the following criteria were met: intubation and mechanical ventilation for at least 48 hours prior to diagnosis; a new or progressive infiltrate on a chest X-ray; purulent tracheobronchial secretions; and Clinical Pulmonary Infection Score (CPIS) more than six [11-14].

Acute pyelonephritis was diagnosed in any patient presenting with all the following: fever, lumbar tenderness or radiological findings consistent with acute pyelonephritis, and pyuria defined as more than $10 \mathrm{WBCs} /$ high power field or positive (+3) dipstick of urine for leukocyte esterase [15].

A diagnosis of intraabdominal infection was made in patients with temperature above $38^{\circ} \mathrm{C}$ or below $36^{\circ} \mathrm{C}$, leukocytosis (WBC >12,000 cells/ $\mu \mathrm{l}$ ) and radiological findings consistent with an intraabdominal infection [15].

Primary bacteremia was defined as any positive blood culture for Gram-positive or Gram-negative microorganisms in the absence of any well-defined focus of infection, including intravascular-access devices [15].

Criteria required for the diagnosis of CAP and HAP included the presence of a new infiltrate on a chest $X$-ray along with two of the following: fever, leukocytosis or leukopenia, and/or purulent sputum. Pneumonia was considered as: CAP whenever the patient did not report any past hospitalization for the past 90 days or stay in a long-term care facility; or HAP when presenting more than 48 hours after hospital admission in any patient not requiring mechanical ventilation [14-16].

Patients were followed up for 28 days. A complete diagnostic work-up was performed comprising history, clinical examination, blood cell counts and biochemistry, blood cultures, chest X-ray, and chest and/or abdominal computed tomography scans if considered necessary. Quantitative cultures of urine or tracheobronchial secretions (TBS) were performed and interpreted as previously described [17] depending on the patient's underlying infection. Within the first 24 hours of the advent of signs of sepsis, $15 \mathrm{ml}$ of heparinized peripheral 
venous blood was sampled after puncture of one forearm vein under sterile conditions.

\section{Laboratory techniques}

For the flow cytometric analysis, red blood cells were lysed with ammonium chloride $1 \mathrm{mM}$ and WBCs were washed three times with PBS (pH 7.2; Merck, Darmstadt, Germany). WBCs were then stained with fluorocolour-conjugated monoclonal antibodies against CD3, CD4, CD8, CD (16+56), CD19 and with the protein annexin- $V$ and propidium iodine (PI) (Immunotech, Marseille, France), and incubated for 15 minutes in the dark. Fluorocolours used were fluorescein isothiocyanate (FITC; emission 525 nm; Immunotech, Marseille, France), phycoerythrin (PE; emission $575 \mathrm{~nm}$; Immunotech, Marseille, France), ECD (emission $613 \mathrm{~nm}$, Immunotech, Marseille, France) and PC5 (emission $670 \mathrm{~nm}$, Immunotech, Marseille, France). The following combinations were applied: antiCD3(FITC)/CD4(PE), anti-CD3(FITC)/CD8(PE), antiCD3(FITC)/CD(16+56)(PE), anti-CD19(FITC), annexin$\mathrm{V}(\mathrm{FITC}) / \mathrm{CD} 4(\mathrm{PE}) / \mathrm{PI}$ (PC5), and annexin-V(FITC)/anti$\mathrm{CD} 8(\mathrm{PE}) / \mathrm{PI}(\mathrm{PC} 5)$. Cells that stained positive for annexin-V and negative for $\mathrm{PI}$ were considered apoptotic.

Flow-cytometric analysis was performed on an EPICS XL/ MSL flow cytometer (Beckman Coulter Co, Miami, FL, USA) with gating for mononuclears based on their characteristic forward and side scattering.

For the isolation of monocytes, blood was layered over Ficoll Hypaque (Biochrom, Berlin, Germany) and centrifuged. Isolated peripheral blood mononuclear cells (PBMCs) were washed three times with PBS $(\mathrm{pH} 7.2)$ and incubated with RPMI 1640 media enriched with 10\% fetal bovine serum (FBS) and $2 \mathrm{mM}$ glutamine, $100 \mathrm{U} / \mathrm{ml}$ penicillin $\mathrm{G}$ and $0.1 \mathrm{mg} /$ $\mathrm{ml}$ streptomycin (Sigma Co, St Louis, MO, USA) in $75 \mathrm{~cm}^{3}$ flasks. After one hour of incubation at $37^{\circ} \mathrm{C}$ in $5 \% \mathrm{CO} 2$, nonadherent cells were removed. Adherent monocytes were thoroughly washed with Hank's solution (Biochrom, Berlin, Germany), harvested with a $0.25 \%$ trypsin/0.02\% ethylenediamine tetraacetic acid (EDTA) solution (Biochrom, Berlin, Germany). Their purity was more than 95\% as defined after staining with anti-CD14 and analysis by a flow cytometer.

Isolated monocytes were counted in a Neubauer plate by trypan blue exclusion of dead cells, distributed in two wells of a 12-well plate and cultured with RPMI 1640 media supplemented with $10 \%$ FBS and $2 \mathrm{mM}$ glutamine with or without the addition of $10 \mathrm{ng} / \mathrm{ml}$ of purified endotoxin (lipopolysaccharide (LPS)) derived from Escherichia coli O155:H5 (Sigma $\mathrm{Co}$, St Louis, MO, USA). After incubation for 24 hours at $37^{\circ} \mathrm{C}$ in a $5 \% \mathrm{CO} 2$ atmosphere, supernatants were collected and stored at $-70^{\circ} \mathrm{C}$ until assayed for cytokines.

Estimation of TNF $\alpha$ and IL- 6 in supernatants was performed by an ELISA (Diaclone, Paris, France). Lowest detection limits were $15.75 \mathrm{pg} / \mathrm{ml}$ for TNF $\alpha$ and $6.25 \mathrm{pg} / \mathrm{ml}$ for IL-6. Concentrations were adjusted as $\mathrm{pg} / 10^{4}$ live cells.

In an attempt to explain our findings, PBMCs of healthy volunteers were exposed to isolates of TBS from patients with VAP and to blood isolates of patients with bloodstream infections enrolled in this study. Current theories attribute pathogenesis of VAP to the aspiration of microbes colonizing the oropharynx in the lower respiratory tract. According to the theories, bacteria replicate gradually and when their growth surpasses a certain threshold then VAP develops $[18,19]$. In an attempt to reproduce the above sequence of events in vitro, PBMCs were isolated from five healthy volunteers as described above. They were distributed in wells of a 12-well plate in RPMI 1640 media supplemented with $10 \%$ FBS and 2 mM glutamine, 100 $\mathrm{U} / \mathrm{ml}$ penicillin $\mathrm{G}$ and $0.1 \mathrm{mg} / \mathrm{ml}$ streptomycin (Sigma Co, St Louis, MO, USA). These PBMCs were stimulated by four different isolates: one of Acinetobacter baumannii and another of $P$ seudomonas aeruginosa isolated at a count of $1 \times 10^{6} \mathrm{cfu} /$ $\mathrm{ml}$ or more from TBS of two different patients with VAP; and one of $A$. baumannii and another of $P$. aeruginosa isolated from blood of two different patients with bacteremia. All isolates were grown for 12 hours in Mueller-Hinton broth (Oxoid Ltd, London, UK) in a shaking-water bath at $37^{\circ} \mathrm{C}$. Then a logphase inoculum of $5 \times 10^{7} \mathrm{cfu} / \mathrm{ml}$ was prepared in MuellerHinton broth using the 0.5 standard of the McFarland climax. Appropriate amounts of that inoculum were used for cell stimulation in four different patterns, as follows.

Pattern A was non-stimulated PBMCs incubated for 4.75 hours in growth medium at $37^{\circ} \mathrm{C}$ in $5 \% \mathrm{CO} 2$.

Pattern B was sequential stimulation in three steps mimicking pathogenesis of VAP. In the first step, PBMCs were exposed for 15 minutes at $37^{\circ} \mathrm{C}$ in $5 \%$ CO2 in $1 \times 10^{3} \mathrm{cfu} / \mathrm{ml}$ of each of the VAP pathogens. Then the plate was centrifuged, supernatants were discarded and the cell pellet was dissolved in 2.4 $\mathrm{ml}$ of growth medium. In the second step, the same procedure as in the first step was repeated after two hours. In the third step, after two hours of incubation at $37^{\circ} \mathrm{C}$ in a $5 \% \mathrm{CO} 2$ atmosphere, PBMCs were stimulated with $1 \times 10^{6} \mathrm{cfu} / \mathrm{ml}$ of each of the two pathogens for 30 minutes. These inocula were selected for stimulation in an attempt to generate conditions of bacterial growth similar to those existing in patients with VAP. Then, the plate was centrifuged.

Pattern $\mathrm{C}$ was an abrupt stimulation with VAP pathogens. The first two steps of pattern $B$ were performed but instead of stimulation with $1 \times 10^{3} \mathrm{cfu} / \mathrm{ml}$ inoculum, Mueller-Hinton broth was added in the plates. The third step was repeated as in pattern B.

Pattern D was an abrupt stimulation with pathogens causing bacteremia mimicking the pathogenesis of bacteremia. After incubation for 4.15 hours at $37^{\circ} \mathrm{C}$ in $5 \% \mathrm{CO}_{2}$ PBMCs were 
exposed for 30 minutes to $1 \times 10^{6} \mathrm{cfu} / \mathrm{ml}$ of each of the two pathogens causing bacteremia. Then the plate was centrifuged.

For all the above patterns, after centrifugation of the plate and removal of supernatants, adherent cells were harvested with a 0.25\% trypsin/0.02\% EDTA solution (Biochrom, Berlin, Germany). Flow cytometric analysis of apoptosis was performed after staining collected cells with annexin-V(FITC)/antiCD4(PE)/PI(PC5) and annexin-V(FITC)/anti-CD14(PE)/ $\mathrm{PI}(\mathrm{PC} 5)$. To exclude debris or red blood cells, collected cells were also stained with anti-CD45 (ECD); their purity was more than $95 \%$.

\section{Statistical analysis}

Septic patients were divided in two groups, those with VAP and those suffering from other infections. Results were expressed as means (standard deviation) for parametric variables and as medians (interquartile range) for non-parametric variables. Comparisons of baseline quantitative characteristics between groups were performed by the Student's t-test and of baseline qualitative characteristics by the chi-squared test. Comparisons of non-parametric quantitative characteristics between groups were performed by the Mann-Whitney $U$ test.

Both groups of patients were additionally divided in two subgroups each, depending on the positive response of monocytes to LPS-stimulation with or without TNF $\alpha$ production. A more than five-fold increase of TNF $\alpha$ production following stimulation was considered a positive response. Survival of two subgroups was estimated by Kaplan-Meier analysis; comparisons were performed by the log-rank test.

Apoptosis of each pattern of stimulation of PBMCs was expressed by means (standard error); comparisons were performed by analysis of variance after Bonferroni correction. Any value of $P$ below 0.05 was considered significant.

\section{Results}

Clinical characteristics of patients enrolled in the study are presented in Table 1. Other infections included pyelonephritis (7 patients), primary bacteremia (10 patients), intraabdominal infection (12 patients), CAP (1 patient) and HAP (2 patients). No differences were found between patients with VAP and patients with other infections regarding sex, age, disease severity (Acute Pathophysiology and Chronic Health Evaluation II score), WBC absolute count and differentiate, as well as the use of corticosteroids for the treatment of septic syndrome. More frequent co-morbidities were chronic obstructive pulmonary disease, diabetes mellitus, congestive heart failure and chronic renal failure, but no difference between groups was observed. Among patients who developed VAP only two had initially presented with other infections, namely peritonitis and cholecystitis, and among patients with other infections only one was primarily hospitalized because of an intraabdominal abscess.

Flow-cytometric data of septic patients with VAP compared to those with other infections are shown in Table 2. The absolute number of CD3(+)/CD4(+) cells was significantly lower in patients with VAP than with other infections $(P=0.034)$. Apoptosis of isolated monocytes was increased in VAP compared with other infections $(P=0.007)$.

Cytokine release by monocytes upon stimulation with LPS is shown in Figure 1. Release of both TNF $\alpha$ and IL-6 from monocytes was lower in patients with VAP-related sepsis than with sepsis related to other types of infection.

Kaplan-Meier analysis of survival of patients subgrouped into responders and non-responders after stimulation with LPS revealed that a positive response after stimulation was a detrimental factor affecting survival among patients with sepsis caused by VAP but not in sepsis caused by other infections. More precisely, among patients with VAP-related sepsis, 28day mortality of responders was $25 \%$ compared with $60 \%$ of non-responders $(P=0.045$, Figure 2$)$. Among those with other infections, 28-day mortality of responders was $11.76 \%$ and of non-responders $28.57 \%(P=0.245$, Figure 2$)$.

To exclude the possibility that results may be related to the process of mechanical ventilation, patients with non-VAP related-sepsis were further divided in to two subgroups, those being intubated and those not being intubated. No difference in the percentage of CD3(+)/CD4(+) lymphocytes and in the apoptosis of monocytes was observed between the two subgroups. More precisely, median expression of CD3/CD4 on lymphocytes was $49.60 \%$ and $54.66 \%$, respectively $(P=$ $0.654)$ and median apoptosis of monocytes was $8.29 \%$ and $15.15 \%$, respectively $(P=0.329)$.

The rate of apoptosis of lymphocytes and of monocytes for each pattern of stimulation is shown in Figure 3. Stimulation according to pattern $B$ mimicking pathogenesis of VAP was accompanied by inhibition of apoptosis of CD4-lymphocytes and by induction of apoptosis of CD14-monocytes compared with both patterns $A$ and $D$.

\section{Discussion}

Sepsis is accompanied by dysregulated immune response. Among patients, those with VAP are considered more compromised than others because of the iatrogenic intervention in mechanical lung defenses due to endotracheal intubation $[19,20]$. A recent publication by our group showed that apoptosis of monocytes in patients with VAP may play a considerable role in the final outcome of the patient [8]. However, the point of discussion is whether this innate immune response is a unique characteristic of sepsis related to VAP or even of sepsis not related to VAP. The present study investigated the 
Table 1

\begin{tabular}{|c|c|c|c|}
\hline & VAP & Other infections & $P$ \\
\hline Male/female & $23 / 13$ & $14 / 18$ & 0.099 \\
\hline Age (years) & $68.88(15.57)$ & $64.41(19.76)$ & 0.300 \\
\hline Sepsis/severe sepsis/septic shock & $7 / 22 / 7$ & $15 / 7 / 10$ & 0.411 \\
\hline APACHE II score & $18.25(4.31)$ & $15.33(5.03)$ & 0.200 \\
\hline WBCs $(/ \mu \mathrm{l})$ & $11530(1179)$ & $14530(1469)$ & 0.141 \\
\hline Monocytes & $234(149)$ & $311(49)$ & 0.422 \\
\hline Lymphocytes & $629(896)$ & $685(172)$ & 0.922 \\
\hline Neutrophils & $8299(1342)$ & $9180(1371)$ & 0.642 \\
\hline Use of steroids $(n, \%)$ & 12 (33.33) & $13(28.12)$ & 0.501 \\
\hline \multicolumn{4}{|l|}{ Comorbidities (n, \%) } \\
\hline COPD & $9(25.00)$ & $3(9.38)$ & 0.083 \\
\hline DM & $9(25.00)$ & $6(18.75)$ & 0.439 \\
\hline $\mathrm{CHF}$ & $3(8.33)$ & $7(21.87)$ & 0.206 \\
\hline CRF & $4(11.11)$ & $2(6.25)$ & 0.414 \\
\hline Prior infections (n) & 2 & 1 & \\
\hline \multicolumn{4}{|l|}{ Number of failing organs ( $n$ ) } \\
\hline Two or more & 8 & 11 & \\
\hline Duration of hospitalization (days) & $18.93(20.84)$ & $19.04(27.90)$ & 0.151 \\
\hline Duration of mechanical ventilation (days) & $18.34(21.11)$ & $26.76(29.1)$ & 0.525 \\
\hline \multicolumn{4}{|l|}{ Bacterial causes $(n, \%)$} \\
\hline P. aeruginosa & 12 (33.33) & $4(12.50)$ & \\
\hline A. baumannii & $7(19.44)$ & $5(15.62)$ & \\
\hline K. pneumoniae & $3(8.33)$ & $5(15.62)$ & \\
\hline E. coli & $1(2.77)$ & $5(15.62)$ & \\
\hline Ent. cloacae & $2(5.55)$ & $2(6.25)$ & \\
\hline
\end{tabular}

Values are expressed as means (standard deviation).

$\mathrm{APACHE}=\mathrm{Acute}$ Physiology and Chronic Health Evaluation; $\mathrm{CHF}=$ congestive heart failure; $\mathrm{CRF}=$ chronic renal failure; $\mathrm{COPD}=\mathrm{chronic}$ pulmonary obstructive disease; $\mathrm{DM}=$ diabetes mellitus; $\mathrm{VAP}=$ ventilator-associated pneumonia; $\mathrm{WBC} s=$ white blood cells.

alterations of innate and of adaptive immune responses in patients with sepsis due to VAP in comparison to septic patients with other infections. Every attempt was made to match both groups of patients according to age, sex, disease severity and causative pathogens. The latter were Gram-negative species. It has to be emphasized that in the Greek setting, VAP is mainly caused by Gram-negative pathogens [21].

Flow cytometry analysis revealed two major differences between sepsis due to VAP and sepsis caused by other infections. The first difference is the decrease of CD3(+)/CD4(+) lymphocytes in VAP. Depletion of T-helper lymphocytes in sepsis has already been described and attributed to accelerated apoptosis [22]. In the present study, no difference in the apop- totic rate of T-helper lymphocytes between the two groups of patients was shown.

The second major finding is a considerable increase of apoptosis of monocytes in patients with VAP. As a consequence of that phenomenon, immunoparalysis of monocytes, which occurs normally in sepsis $[23,24]$, is pronounced in VAP compared with other infections. Immunoparalysis was stated by the inability of monocytes to produce sufficient amounts of TNF $\alpha$ and IL- 6 after stimulation with LPS (Figure 1). Among patients with VAP, those with monocytes responding to LPS stimulation presented a survival benefit compared with nonresponders. That was not the scenario for sepsis caused by other types of infection. Although it was obvious that VAP was 
Table 2

Flow-cytometric data of patients with sepsis due to VAP and sepsis caused by other nosocomial infections

\begin{tabular}{|c|c|c|c|}
\hline & VAP & Other infections & $P$ \\
\hline $\mathrm{CD} 3(+) / \mathrm{CD} 4(+)$ & $208.52(192)$ & $280.68(508.7)$ & 0.034 \\
\hline $\mathrm{CD} 3(+) / \mathrm{CD} 8(+)$ & $114.8(123.26)$ & $102.49(244.7)$ & 0.787 \\
\hline $\mathrm{CD} 3(+) / \mathrm{CD}(16+56)(+)$ & $18.09(34.41)$ & $15.11(49.49)$ & 0.940 \\
\hline${ }^{*}$ Natural killer cells & $26.98(62.94)$ & $39.61(45.22)$ & 0.463 \\
\hline CD19(+) & $28.00(70.00)$ & 37.93 (33.82) & 0.219 \\
\hline Annexin(+)/CD4(+)/PI(-) & $3.16(4.48)$ & $2.33(7.64)$ & 0.944 \\
\hline Annexin(+)/CD8(+)/PI(-) & $3.74(8,71)$ & $6.47(15.71)$ & 0.269 \\
\hline Annexin(+)/PI(-) of isolated monocytes & $20.62(28.11)$ & $12.19(22.98)$ & 0.007 \\
\hline
\end{tabular}

*Natural killer cells were defined as CD3(-)/CD(16+56)(+). Values are expressed as median (IQR) absolute numbers for CD3(+)/CD4(+), CD3 $(+) / C D 8(+), C D 3(+) / C D(16+56)(+)$, natural killer and CD19(+) cells and as median (interquartile range) percentages for Annexin $(+) /$ $\mathrm{CD} 4(+) / \mathrm{PI}(-)$, Annexin(+)/CD8(+)/PI(-) and Annexin(+)/PI(-) of isolated monocytes. VAP = ventilator-associated pneumonia.

a situation of profound immunoparalysis, survival was prolonged among those patients with adequate monocyte function (Figure 2).

A question emerging from these results was whether immunoparalysis observed among patients with VAP was a result of their baseline characteristics. The two groups of patients did not differ in sex, age, disease severity or co-morbidities. The use of corticosteroids for the treatment of the septic syndrome was also similar between VAP and non-VAP septic patients. The presence of prior bacterial infections was rare in both groups. The possibility that mechanical ventilation could have acted as a confounding factor was excluded, because no difference was observed when the percentages of T-helper lymphocytes and the apoptosis of monocytes between intubated and non-intubated non-VAP patients were compared. $P$. aeruginosa and $A$. baumannii were more frequently responsible for VAP than for other infections. This was expected because these two microorganisms constitute the two major pathogens of nosocomial pneumonia in Greece [25].

In vitro findings support the hypothesis that one major cause of immune alterations in patients with sepsis is the type of contact of immune cells with the pathogens. More precisely, in

Figure 1
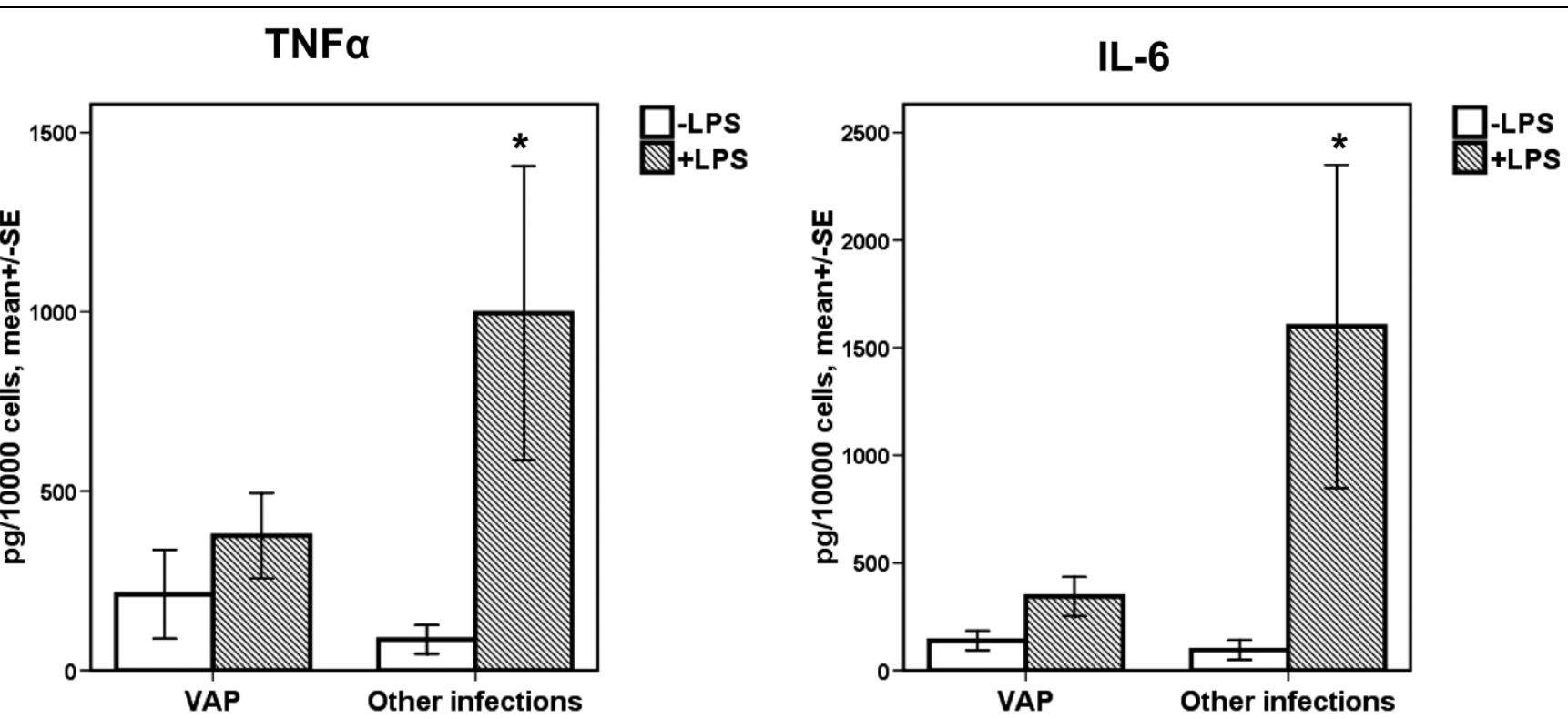

TNF $\alpha$ and IL- 6 production from the supernatants of monocytes. Concentrations of TNF $\alpha$ and IL- 6 of supernatants of monocytes of patients with sepsis due to ventilator-associated pneumonia (VAP) and patients with sepsis caused by other nosocomial infections. The asterisk denotes significant difference between the two groups of patients. $(P=0.008$ for TNF $\alpha ; P=0.003$ for IL-6). LPS $=$ lipopolysaccharide; SE $=$ standard error. 

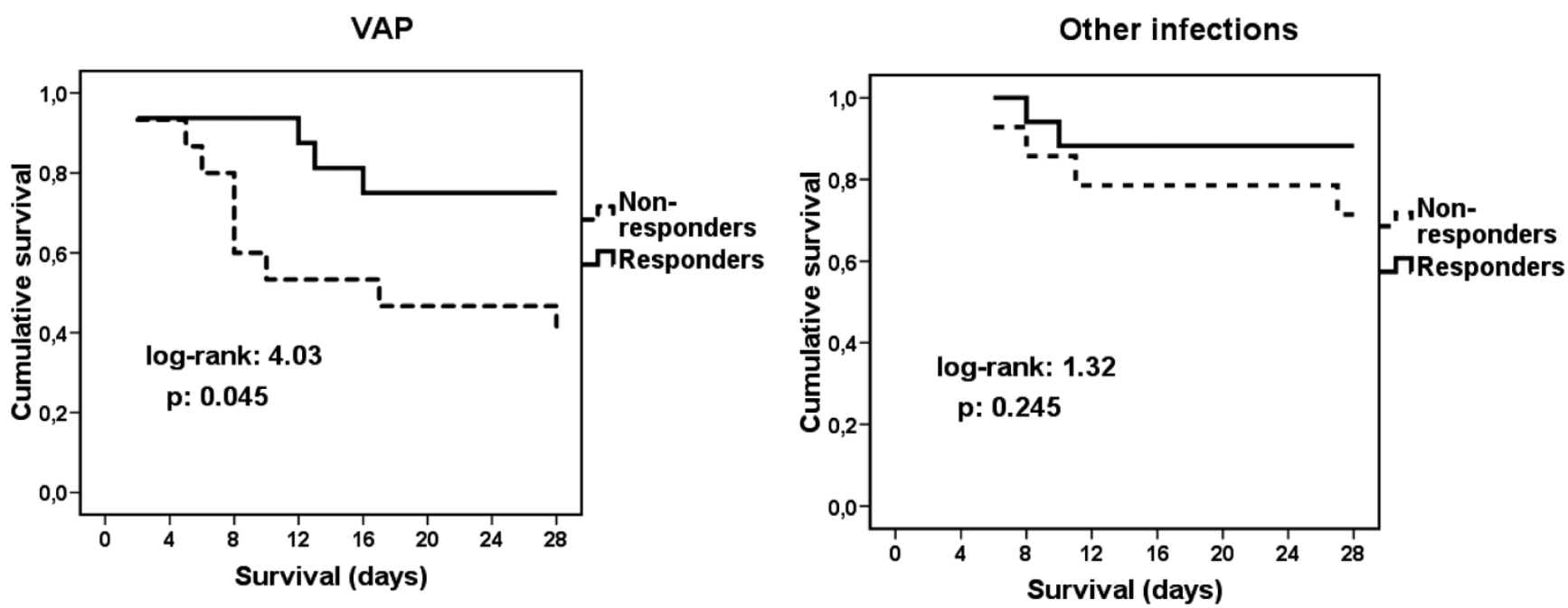

Comparison of survival of septic patients. Comparison of survival of septic patients due to ventilator-associated pneumonia (VAP) and patients with sepsis caused by other infections depending on the presence or absence of response of their monocytes to stimulation with lipopolysaccharide.

patients with VAP the immune system is gradually exposed to the pathogen. The latter is entering the airways through aspiration of the oropharyngeal flora and then steadily increases to an amount able to induce VAP. As a consequence, the immune system is gradually exposed to sequentially increased bacterial inocula, which leads to decreased apoptosis of CD4lymphocytes and to increased apoptosis of CD14-monocytes (Figure 3, pattern B). When VAP evolves abruptly, similar alter- ations are not seen (Figure 3, pattern C). This is also the case with bacteremia (Figure 3, pattern D).

The in vitro experiment was based on the assumption that VAP supervenes as a result of gradual and continuous exposure of the innate immune system to the pathogen while non-VAP sepsis is the result of an abrupt stimulation of the innate immune system. The response of PBMCs of healthy volunteers may dif-

Figure 3
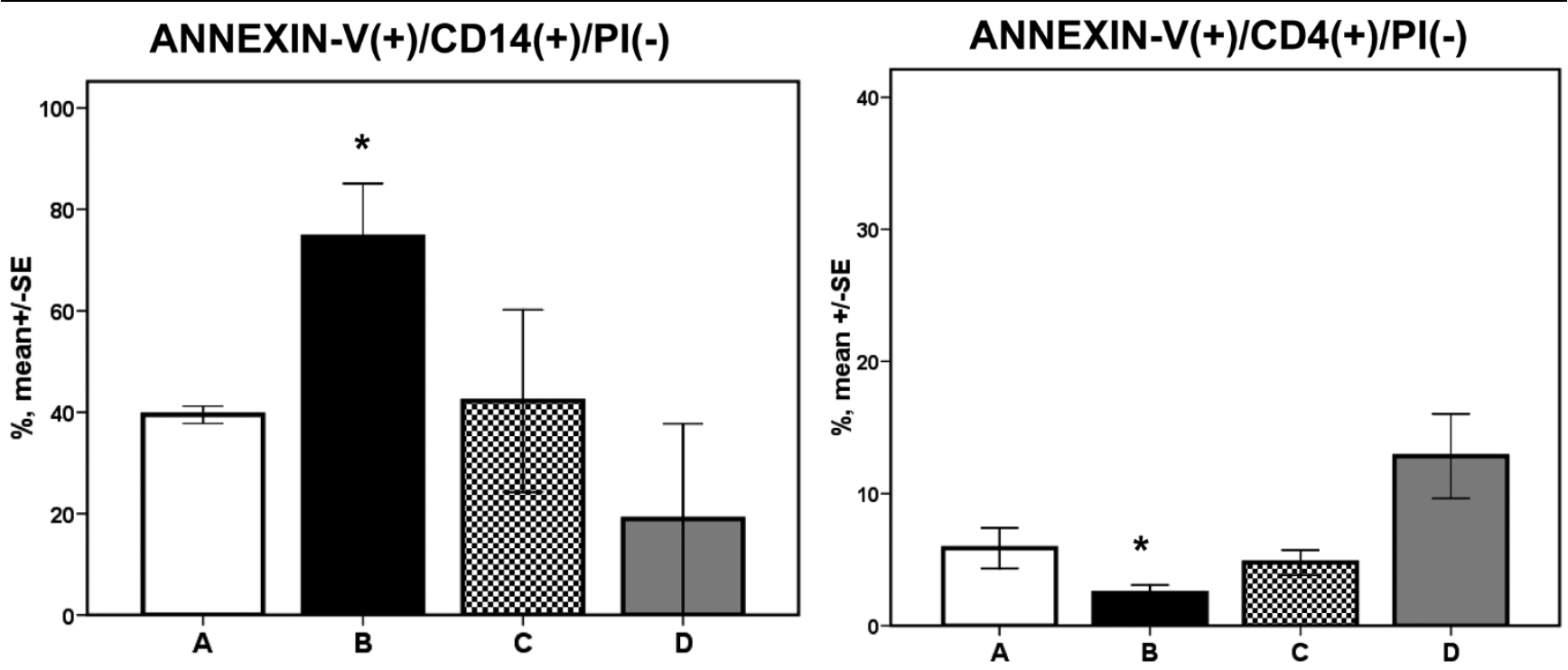

Apoptosis of CD14-monocytes and of CD4-lymphocytes of healthy volunteers. Induction of apoptosis of CD14-monocytes and inhibition of apoptosis of CD4-lymphocytes of healthy volunteers according to four different patterns of stimulation by isolates of Acinetobacter baumannii and of $P$ seudomonas aeruginosa. $\mathrm{A}=$ un-stimulated controls; $\mathrm{B}=$ three-step stimulation mimicking pathogenesis of ventilator-associated pneumonia (VAP); $\mathrm{C}=$ abrupt stimulation with pathogens of VAP; and $D=$ abrupt stimulation mimicking pathogenesis of bacteremia. Asterisks denote significant difference between patterns $B$ and $D$ and between patterns $B$ and $A . S E=$ standard error. 
fer from those of PBMCs of septic patients. A number of factors participate to the interactions between bacteria and the immune system, such as virulence genes or pattern recognition receptors, whose role was not studied in our setting. Further investigation is mandatory in order to clarify our hypothesis about the pathogenesis of VAP.

\section{Conclusions}

The presented findings reveal that innate and adaptive immune responses differ considerably between sepsis due to VAP and sepsis due to other types of nosocomial infection. VAP is characterized by substantial decrease of CD4-lymphocytes and immunoparalysis of monocytes in contrast to other infections. The mechanism of bacterial pathogenesis of VAP may help explain these differences. The latter could constitute a novel therapeutic target for the management of the septic patient with VAP.

Key messages
Sepsis due to VAP is characterized by decrease of
CD3/CD4(+) lymphocytes and immunoparalysis of
monocytes compared with sepsis caused by other
nosocomial infections.
The mechanism of bacterial pathogenesis of VAP
seems to play a crucial role in the explanation of these
differences.

\section{Competing interests}

The authors declare that they have no competing interests.

\section{Authors' contributions}

AP participated in the follow-up of patients, performed the in vitro experiments and the estimation of TNF $\alpha$ and IL-6, participated in the immunophenotypic analysis, analysed the data and wrote the manuscript. IT participated in the enrolment and follow-up of patients. AK and VK participated in the immunophenotypic analysis. $H G$ and $A A$ drafted the manuscript. EJG-B participated in the study design and the analysis of data and drafted the manuscript.

\section{References}

1. Vincent J-L: Clinical sepsis and septic shock-definition, diagnosis and management principles. Langenbecks Arch Surg 2008, 393:817-824

2. Jean-Baptiste E: Cellular mechanisms in sepsis. J Intensive Care Med 2007, 22:63-72.

3. Alberti C, Brun-Buisson C, Buchardi H, Martin C, Goodman S, Artigas A, Sicignano A, Palazzo M, Moreno R, Boulmé R, Lepage E, Le Gall J: Epidemiology of sepsis and infection in ICU patients from an international multicentre cohort study. Intensive Care Med 2002, 28:108-121.

4. Chastre J: Ventilator-associated pneumonia: what is new? Surg Infect (Larchmt) 2006, 7(Suppl 2):S81-85.

5. Davis KA: Ventilator-associated pneumonia: a review. J Intensive Care Med 2006, 21:211-226.

6. Augustyn B: Ventilator-associated pneumonia, risk factors and prevention. Crit Care Nurse 2007, 27:32-40.
7. Hunter J, Annadurai S, Rothell M: Diagnosis, management and prevention of ventilator-associated pneumonia in the UK. Eur $J$ Anaesthesiol 2007, 24:971-977.

8. Giamarellos-Bourboulis EJ, Routsi C, Plachouras D, Markaki V, Raftogiannis M, Zervakis D, Koussoulas V, Orfanos S, Kotanidou A, Armaganidis A, Roussos C, Giamarellou H: Early apoptosis of blood monocytes in the septic host: is it a mechanism of protection in the event of septic shock? Crit Care 2006, 10:146-154.

9. The ACCP/SCCM Consensus Conference Committee, American College of Chest Physicians/Society of Critical Care Medicine: Definitions for sepsis and organ failure and guidelines for the use of innovative therapies in sepsis. Chest 1992, 101:1644-1655

10. Levy M, Fink M, Marshall J, Abraham E, Angus D, Cook D, Cohen J, Opal S, Vincent J, Ramsay G, SCCM/ESICM/ACCP/ATS/SIS: 2001 SCCM/ESICM/ACCP/ATS/SIS International Sepsis Definitions conference. Crit Care Med 2003, 31:1250-1256.

11. Baughman R: Diagnosis of ventilator-associated pneumonia. Curr Opin Crit Care 2003, 9:397-402.

12. Rea-Neto $A$, Youssef N, Tuche F, Brunkhorst F, Ranieri M, Reinhart $\mathrm{K}$, Sakr Y: Diagnosis of ventilator-associated pneumonia: a systematic review of the literature. Crit Care 2008, 12:R56.

13. Soto G: Diagnostic strategies for nosocomial pneumonia. Curr Opin Pulm Med 2007, 13:186-191.

14. Rello J, Paiva J, Baraibar J, Barcelinna F, Bodi M, Castander D, Correa H, Diaz E, Garnacho J, Llorio M, Rios M, Rodriguez A, SolèViolán : International conference for the Development of Consensus on the Diagnosis and treatment of Ventilator-Associated Pneumonia. Chest 2001, 120:955-970.

15. Calandra T, Cohen J: The International Sepsis Forum Consensus Conference on Definitions of Infection in the Intensive Care Unit. Crit Care Med 2005, 33:1538-1548.

16. Kollef $\mathrm{MH}$ : What is ventilator-associated pneumonia and why is it important? Respir Care 2005, 50:714-724.

17. Camargo LFA, De Marco FV, Barbas CSV, Hoelz C, bueno MA, Rodriguez M Jr, Amado VM, Caserta R, Martino MD, Pasternak J, Knobel E: Ventilator associated pneumonia: comparison between quantitative and qualitative cultures of trachea aspirates. Crit Care 2004, 8:R422-R430.

18. Crnich C, Safdar N, Maki D: The role of intensive care unit environment in the pathogenesis and prevention of ventilatorassociated pneumonia. Respiratory Care 2005, 50:813-838.

19. Safdar N, Crnich C, Maki D: The pathogenesis of ventilatorassociated pneumonia: its relevance to developing effective strategies for prevention. Respiratory Care 2005, 50:725-741.

20. Wunderlink R: Nosocomial pneumonia, including ventilatorassociated pneumonia. Proc Am Thorac Soc 2005, 2:440-444.

21. Giamarellos-Bourboulis EJ, Pechère JC, Routsi C, Plachouras D, Kollias S, Raftogiannis M, Zervakis S, Baziaka F, Koronaios A, Antonopoulou A, Markaki V, Koutoukas P, Papadomichelakis E, Tsaganos T, Armaganidis A, Koussoulas V, Kotanidou A, Roussos $\mathrm{C}$, Giamarellou $\mathrm{H}$ : Effect of clarithromycin in patients with sepsis and ventilator-associated pneumonia. Clin Infect Dis 2008, 46:1157-1164.

22. Hotchkiss R, Tinsley K, Swanson P, Schmieg R, Hui J, Chang K, Osborne D, Freeman B, Cobb P, Buchman T, Karl I: Sepsisinduced apoptosis causes progressive profound depletion of $\mathrm{B}$ and $\mathrm{CD4}^{+} \mathrm{T}$ lymphocytes in humans. $J$ Immunol 2001, 166:6952-6963.

23. Williams M, Withington S, Newland A, Kelsey S: Monocyte anergy in septic shock is associated with a predilection to apoptosis and is reversed by granulocyte-macrophage colony-stimulating factor ex vivo. J Infect Dis 1998, 178:1421-1423.

24. Haveman JW, Kobold AC, Tervaert JW, Berg AP van den, Tulleken $\mathrm{JE}$, Kallenberg CGM, The TH: The central role of monocytes in the pathogenesis of sepsis: consequences for immunomonitoring and treatment. Neth J Med 1999, 55:132-141.

25. Koulenti D, Lisboa T, Brun-Buisson C, Krueger W, Macor A, SoleViolan J, Diaz E, Topelli A, DeWaele J, Carneiro A, Martin-Loeches $M$, Armaganidis A, Rello J: Spectrum of practice in the diagnosis of nosocomial pneumonia in patients requiring mechanical ventilation in European intensive care units. Crit Care Med 2009, 37:2360-2368. 